(Aus dem psychol. Seminar der Universität Berlin.)

\title{
Studien über Unterbrechungstöne.
}

\author{
Erste Mittheilung. \\ Von
}

Karl L. Schaerer und Otto Abraham.

I.

Wenn ein einfacher Ton, dessen Schwingungszahl $m$ ist, $n$ Mal in der Secunde intermittirt, so hört man bekanntlich unter geeigneten Umständen neben dem Hauptton $m$ noch einen Intermittenzoder Unterbrechungston von der Tonhöhe $n$.

Um Unterbrechungstöne hervorzubringen, hat man sich verschiedener Methoden bedient, deren einfachste die von Dennert in seinen „Akustisch-physiologischen Untersuchungen "1) beschriebene ist. Dieser Autor liess ,auf einer Scheibe mit 3 Kreisen, welche in 96 gleiche Theile getheilt waren, die Löcher so ausschlagen, dass an den Theilungen immer alternirend auf dem ersten Kreise 4, auf dem zweiten 3 und auf dem dritten Kreise 2 Löcher ausgeschlagen wurden, während der anstossende Raum für die gleiche Anzahl Löcher undurchschlagen blieb, so dass also auf dem ersten Kreise 12 Piècen à 4 Löcher, auf dem zweiten 16 Piècen à 3 Löcher und auf dem dritten 24 Piècen à 2 Löcher vorhanden waren. Wurden nun diese Kreise während der Rotation der Scheibe angeblasen, so hörte man bei langsamer Umdrehung Stösse, die bei schnellerer Umdrehung in Töne übergingen, und zwar zuerst die Stösse der 24 Piècen, dann die der 16 und zuletzt die der 12 Piècen. Die Töne standen in jeder Phase des Versuches im Verhältniss von $1:{ }^{4} / 3: 2$, so dass man, wenn der tiefste der 3 Töne die Höhe des kleinen $c$ erreicht hatte, die 3 Töne in der Reihenfolge $c, f, c^{1}$ neben dem Ton $c^{3}$, welcher der Eintheilung von 96 entsprach, hörte".

1) Archiv für Ohrenheilkunde Bd. 24, S. 181. 1887. 
Den so erzeugten Unterbrechungstönen liegen, wie den Unterbrechungstönen überhaupt, nach Ansicht derjenigen Autoren, die sich bisher mit Unterbrechungstönen beschäftigt haben, nicht etwa pendelförmige Schwingungen der Luft zu Grunde. Sie sollen vielmehr subjectiv im Obre aus den Intermittenzen des Haupttones entstehen.

Der Eine von uns hat nun bereits bei früherer Gelegenbeit ${ }^{1}$ ) auf die Nothwendigkeit hingewiesen, über die Frage, ob die Unterbrechungstöne objective oder subjective Töne sind, genauere Untersuchungen anzustellen, und es ist der Zweck dieser Abhandlung, einige unserer diesen Gegenstand betreffenden Beobachtungen mitzutheilen.

Wir haben zunächst die oben erwähnten Experimente Dennert's wiederholt, wozu wir eine mit mehreren Löcherkreisen versehene kreisförmige, $4 \mathrm{~mm}$ dicke, bölzerne Scheibe von $15 \mathrm{~cm}$ Radius benutzten. Der Durchmesser der Löcher betrug ebenso wie der Abstand derselben von einander $5 \mathrm{~mm}$. In einem der Kreise, welcher aus 88 Löchern bestand, wurden 44 derselben mit kleinen, gut passenden Korkstöpselchen abgedichtet, und zwar so, dass immer vier verschlossene mit vier offenen Löchern abwechselten. Die Scheibe wurde dann durch einen hinreichend constant laufenden Elektromotor in Rotation versetzt und die Löcherreihe angeblasen.

Der erste Versuch ergab als Hauptton $c^{4}$ und als Unterbrechungston $c^{1}$. Die Controle der Tonhöhen wurde uns dadurch wesentlich erleichtert, dass der eine von uns (Abraham) über ein absolutes Gehör von ausserordentlicher Sicherheit verfügt, welches ihn befähigt, die Höhe jedes Tones sofort angeben zu können. Der Unterbrechungston war deutlich überall in der Umgebung der Scheibe zu hören und wurde durch einen gleichgestimmten, in das Ohr eingeführten cylindrischen Resonator sehr merklich verstärkt, wovon ausser uns auch Herr Prof. C. Stumpf sich überzeugt hat. Die Verstärkung blieb aus, wenn statt der Reihe 88 eine der anderen angeblasen wurde, oder wenn man den Unterbrechungston mit irgend einem anderen Resonator auscultirte.

Durch Veränderungen der Rotationsgeschwindigkeit des Elektromotors liess sich die Höhe des Haupt- und Unterbrechungstones innerhalb gewisser Grenzen variiren; aber auch in diesen Fällen

1) Karl L. Schaefer, Eine neve Erklärung der subjectiven Combinationstöne etc. Dieses Archiv Bd. 78, S. 512. 
wurde der Unterbrechungston durch seinen Resonator, und nur durch ihn, verstärkt. Die Zunahme der Intensität im Resonator war stets so erheblich, dass jede Täuschung ausgeschlossen war. Es wurde vielmehr wiederholt an dem plötzlichen Versagen des Resonators zuerst erkannt, wenn die Geschwindigkeit der Scheibe und damit die Höhe des Unterbrechungstones sich geändert hatte. Eine noch instructivere und zugleich zur Demonstration vor einem grösseren Auditorium geeignete Form des Versuches besteht darin, den Resonator des Unterbrechungstones der Anblaseröhre gegenüber dicht an die rotirende Scheibe $\mathrm{zu}$ halten. Der Unterbrechungston wird dadureh mächtig verstärkt. Diese Wirkung tritt andererseits nicht ein, wenn man einen falschen Resonator nimmt oder eine andere Reihe anbläst.

In einem Falle, wo die Schwingungszahl des Unterbrechungstones etwa 300 Schwingungen betrug, näherten wir den hölzernen Resonanzkasten einer Gabel von etwa 300 Schwingungen der Scheibe. Auch dadurch wurde der Unterbrechungston erheblich lauter. Ferner konnte der erste Oberton des Unterbrechungstones auf die gleiche Weise durch den entsprechenden Resonator verstärkt werden. Hieraus folgt, dass der "Unterbrechungston" kein einfacher Ton ist, sondern ein Klang, wofür überdies auch seine Klangfarbe spricht.

Nach diesen Versuchen unterliegt es keinem Zweifel, dass durch das Anblasen eines rotirenden, mit Unterbrechungendurchsetzten Löcherkreises ein objectiver, auf pendelförmigen Schwingungen der Luft beruhender Ton, dessen Schwingungszahl mit der Anzahl der Unterbrechungen übereinstimmt, nebst Obertönen zu Stande kommen kann.

II.

Rudolph Koenigi) hat bei seinen Versuchen über Intermittenztöne auch Sirenenscheiben benutzt, auf welchen die Löcher gleichen Abstand von einander hatten, aber periodisch an Grösse zuund abnahmen, so dass also ein Ton von periodisch wechselnder Intensität in das Ohr des Beobachters gelangte, wenn die Löcher mit einer Röhre von dem Durchmesser der grössten Löcher angeblasen wurden. Es wurde hierbei ein Ton gehört, dessen Schwingungs-

1) Quelques expériences d'acoustique p. 140 f. Paris 1882. 
zahl mit der Anzahl der periodischen Intensitätsschwankungen in einer Secunde übereinstimmte.

Dennert konnte diese Thatsache bestätigen. Er theilt darüber ${ }^{1}$ ) Folgendes mit: „Eine Sirene mit drei Kreisen von 96 gleich abstehenden Löchern, welche auf dem ersten $16 \mathrm{Mal}$ von $1-6 \mathrm{~mm}$ zu- und abwehmen, auf dem zweiten $12 \mathrm{Mal}$ und auf dem dritten $8 \mathrm{Mal}$, wurde mit einer Röhre von $6 \mathrm{~mm}$ Durchmesser angeblasen. Man hörte nun auf allen drei Kreisen bei langsamer Umdrehung Stösse, die bei zunehmender Rotationsgeschwindigkeit in Töne übergingen. Die drei resultirenden Töne stehen in jeder Phase des Versuches, ganz entsprechend den 16, 12 und 8 Löcherperioden, im Verhältniss von $1: 3 / 2: 2 \mathrm{zu}$ einander, so dass also, wenn der tiefste der drei Töne, der den acht Löcherperioden entsprechende Ton, die Höhe des $C$ der grossen Octave erreicht hat, die drei Töne $C, G, c$ neben $g^{2}$, dem Tone, welcher den 96 Löchern in dieser Phase des Versuches entspricht, gehört werden. Man kann sich nun auch durch folgenden Controlversuch überzeugen, dass die drei resultirenden Töne in Bezug auf ihre Schwingungszahl in jeder Phase des Versuches der Anzahl der Löcherperioden entsprechen. Wenn man nämlich auf Kreisen unterhalb der Kreise mit den Löcherperioden und entsprechend den einzelnen Perioden ebenso viele Löcher anbringen lässt, also entsprechend den 16,12,8 Löcherperioden 16, 12, 8 Löcher und nun abwechselnd einmal die Kreise mit den Löcherperioden und dann die Controlkreise anbläst, so wird man sich sehr leicht überzeugen, dass einmal die drei Töne jeder der beiden Tongruppen unter einander genau in demselben Verhältniss zu einander stèhen und dass zweitens auch die correspondirenden Töne dieselbe Tonlage haben."

Herr Geh. Sanitätsrath Dennert hatte die Güte, uns unter vielen anderen auch die bier beschriebene, nach den Angaben Koenig's verfertigte Scheibe zur Verfügung zu stellen. Dieselbe lässt die in Rede stehenden Intermittenztöne mit grosser Deutlichkeit hören, so dass die Prüfung der letzteren auf ihre Objectivität keinerlei Schwierigkeiten begegnet. Wir gaben der Scheibe zunächst eine solche Geschwindigkeit, dass die äusserste Reihe nebst der zugehörigen Controlreihe den Ton $c^{1}$ ergab. Ein auf diese Tonhöhe abgestimmter cylindrischer Resonator aus Blech verstärkte gegen die

1) a. a. $0.5 \cdot 179$ 
Scheibe gehalten den Ton erheblich. Auch der Ton der entsprechenden Controlreibe wurde bei Annäherung des Resonators lauter. Dagegen blieb der Resonator stumm, sobald eine der anderen Reihen tönte. Die nämlichen Resultate erhielten wir mit einem hölzernen Resonanzkasten. Wird der Resonator für den Ton $c^{1}$ ins Obr eingefügt, so erklingt der Ton $e^{1}$ klar und deutlich in demselben dann und nur dann, wenn die äusserste Reihe angeblasen wird. Diese Versuche, die sich durch Veränderungen der Rotationsgeschwindigkeit beliebig variiren lassen, ergeben, dass auch der aus dem periodischen Grössenwechsel der Sirenenlöcher resultirende "Intermittenzton" objectiv als pendelförmige Componente in der durch das Anblasen einer solchen Löcherreihe entstehenden Klangwelle enthalten ist. 